\title{
QUASI-SIMILAR MODELS FOR NILPOTENT OPERATORS $\left({ }^{1}\right)$
}

BY

\author{
C. APOSTOL, R. G. DOUGLAS \\ AND C. FOIAS
}

\begin{abstract}
Every nilpotent operator on a complex Hilbert space is shown to be quasi-similar to a canonical Jordan model. Further, the para-reflexive operators are characterized generalizing a result of Deddens and Fillmore.
\end{abstract}

A familiar result states that each nilpotent operator on a finite dimensional complex Hilbert space is similar to its adjoint. One proof proceeds by showing that both a nilpotent operator and its adjoint have the same canonical form. In this note we show that although this result does not extend to infinite dimensional spaces, the weaker quasi-similarity version of it, together with the proof indicated above, still holds on any Hilbert space. This yields an affirmative answer to a question raised by $P$. Rosenthal in connection with the content of [3].

The canonical form exhibited provides positive evidence that the theory of Jordan models might be extended to cover operators of class $C_{0}$ of infinite multiplicity and indeed, considerable progress [2] has been made recently in this direction. Although the Jordan model for nilpotent operators on infinite dimensional Hilbert spaces is no longer unique, we single out a "canonical" model. A similar result has been obtained independently by Berkovici [1]. We conclude with an application of our results to extend to infinite dimensional spaces a theorem of Deddens and Fillmore [4] which characterizes reflexive operators on finite dimensional spaces.

We want to thank Lawrence Williams for pointing out an error in an earlier version of this note.

1. In this note, a nilpotent operator $T$ will be called a Jordan operator if $T=\bigoplus_{\alpha} T_{\alpha}$, where each $T_{\alpha}$ operates on some $C^{l}$ for $0<l_{\alpha}<\infty$ by the Jordan one-cell matrix

Received by the editors October 24, 1975.

AMS (MOS) subject classifications (1970). Primary 47 B99; Secondary 47A15, 47 A45.

(1) Research partially supported by a grant from the National Science Foundation.

Copyright 1977 , American Mathematical Society 


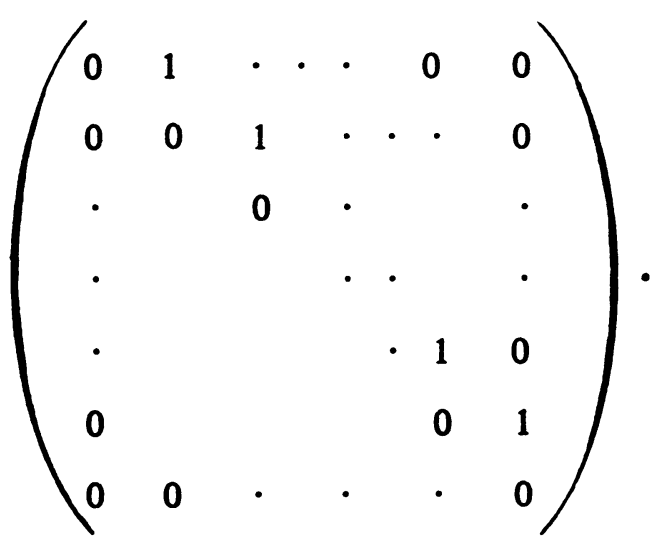

Recall that an operator $X$ between Hilbert spaces $H$ and $K$ is said to be a quasi-affinity if $\operatorname{ker} X=(0)$ and $\operatorname{clos}(X H)=K$. An operator $A$ on $H$ is said to be a quasi-affine transform of an operator $B$ on $K$ if there exists a quasi-affinity $X$ such that $X A=B X$. Finally, two operators $A$ and $B$ are quasi-similar if each is a quasi-affine transform of the other. For further information on these concepts see the monograph [8, Chapter II, No. 3.2], or [7].

Our main result is given by the following

THEOREM 1. Every nilpotent operator $T$ is quasi-similar to a Jordan operator $T_{0}$.

Since for any Jordan operator $T_{0}$, the operators $T_{0}$ and $T_{0}^{*}$ are obviously unitarily equivalent, we can infer

THEOREM 2. If $T$ is a nilpotent operator, then $T$ and $T^{*}$ are quasi-similar.

Before starting the proof of Theorem 1, we give an example to show that quasi-similarity cannot be replaced by similarity.

Let $X$ be any compact quasi-affinity on an infinite dimensional Hilbert space $H$ (for example, the Volterra operator on $L^{2}(0,1)$ ) and consider the operator $T$ defined by

$$
T=\left(\begin{array}{lll}
0 & X & 0 \\
0 & 0 & I \\
0 & 0 & 0
\end{array}\right) \quad \text { on } H \oplus H \oplus H .
$$

Clearly $T^{3}=0$ and thus $T$ and $T^{*}$ are quasi-similar by Theorem 2, but $T$ and $T^{*}$ are not similar. $\left({ }^{2}\right)$ The proof of this is straightforward.

If $S$ were an invertible operator on $H \oplus H \oplus H$ with matrix

(2) The same example was found independently by $H$. Radjavi (see $[3, \S 6])$. 


$$
S=\left(\begin{array}{ccc}
A_{0} & B_{0} & C_{0} \\
A_{1} & B_{1} & C_{1} \\
A_{2} & B_{2} & C_{2}
\end{array}\right)
$$

which satisfied $S T^{*}=T S$, then a simple computation shows that $B_{2}=C_{2}=C_{1}$ $=0, C_{0}=X B_{1}$ and $A_{2}=B_{1} X^{*}$. Thus the operator

$$
S_{0}=\left(\begin{array}{ccc}
A_{0} & B_{0} & 0 \\
A_{1} & B_{1} & 0 \\
0 & 0 & 0
\end{array}\right)
$$

is a compact perturbation of $S$, and hence a Fredholm operator, which is contradicted by the fact that $\operatorname{ker} S_{0}=(0) \oplus(0) \oplus H$ is not finite dimensional (cf. [5, Chapter 5]).

2. We start the proof of Theorem 1 with the following

LEMMA 1. If $T_{0}$ and $T_{1}$ are two Jordan operators and $T_{0}$ is a quasi-affine transform of $T_{1}$, then $T_{0}$ and $T_{1}$ are quasi-similar.

Proof. The fact that $T_{0}$ is a quasi-affine transform of $T_{1}$ means that there exists a quasi-affinity $X$ such that $X T_{0}=T_{1} X$ and thus $T_{0}^{*} X^{*}=X^{*} T_{1}^{*}$. Since $T_{i}$ is a Jordan operator, there exists a unitary operator $U_{j}$ such that $T_{j}^{*}=$ $U_{j}^{*} T_{j} U_{j}(j=0,1)$. Therefore, $T_{0}\left(U_{0} X^{*} U_{1}^{*}\right)=\left(U_{0} X^{*} U_{1}^{*}\right) T_{1}$, where $U_{0} X^{*} U_{1}^{*}$ is a quasi-affinity, and $T_{1}$ is also a quasi-affine transform of $T_{0}$. Consequently, $T_{0}$ and $T_{1}$ are quasi-similar.

LEMMA 2. Any nilpotent operator $T$ has a quasi-affine transform $T_{0}$ which is a Jordan operator.

Proof. Suppose that $T^{n}=0, T^{n-1} \neq 0$ for some $n \geqslant 1$. If we set

$$
\begin{aligned}
& X_{j}=\operatorname{ker} T^{j} \ominus \operatorname{ker} T^{j-1} \text { for } j=1,2, \ldots, n \text {, } \\
& y_{n}=X_{n}, \quad y_{n-1}=X_{n-1} \cap\left(T Y_{n}\right)^{\perp}, \ldots, \\
& y_{1}=X_{1} \cap\left(T^{n-1} y_{n}+\cdots+T y_{2}\right)^{\perp} \text { and } \\
& H_{0}=\underbrace{\left(y_{n} \oplus \cdots \oplus y_{n}\right)}_{n \text { times }} \oplus \frac{\left(y_{n-1} \oplus \cdots \oplus y_{n-1}\right)}{(n-1) \text { times }} \oplus \oplus\left(y_{2} \oplus y_{2}\right) \oplus y_{1}
\end{aligned}
$$

we can define the bounded operators $T_{0}$ on $H_{0}$ and $A: H_{0} \rightarrow H$ by the equations 


$$
\begin{aligned}
T_{0}\left(y_{n}^{1} \oplus \cdots \oplus y_{n}^{n} \oplus \cdots \oplus y_{2}^{1} \oplus y_{2}^{2} \oplus y_{1}^{1}\right) \\
\quad=0 \oplus y_{n}^{1} \oplus \cdots \oplus y_{n}^{n-1} \oplus \cdots \oplus 0 \oplus y_{2}^{1} \oplus 0
\end{aligned}
$$

and

$$
\begin{aligned}
A\left(y_{n}^{1}\right. & \left.\oplus \cdots \oplus y_{n}^{n} \oplus \cdots \oplus y_{2}^{1} \oplus y_{2}^{2} \oplus y_{1}^{1}\right) \\
& =y_{n}^{1}+\cdots+T^{n-1} y_{n}^{n}+\cdots+y_{2}^{1}+T y_{2}^{2}+y_{1}^{1}
\end{aligned}
$$

It is easy to see that $T_{0}$ is a Jordan operator and that $A T_{0}=T A$. Using the fact that

$$
\begin{aligned}
\left(y_{1}+\cdots+T^{n-1} y_{n}+y_{2}+\cdots+T^{n-2} y_{n}\right. & +\cdots+y_{k} \\
& \left.+\cdots+T^{n-k} y_{n}\right)^{-}=\operatorname{ker} T^{k},
\end{aligned}
$$

which is proved by induction on $k$, we conclude that $\operatorname{clos}\left(A H_{0}\right)=H$. To complete the proof we must show that $A$ is injective.

If $A$ is not injective, there must exist $y_{j}^{k}$ in $y_{j}, 1 \leqslant j \leqslant n, 1 \leqslant k \leqslant j$, such that

$$
\sum_{j=1}^{n} \sum_{k=1}^{j} T^{k-1} y_{n-j+k}^{k}=0 \text { but } \sum_{j=1}^{n} \sum_{k=1}^{j}\left\|y_{n-j+k}^{k}\right\| \neq 0 .
$$

Let $m$ be the smallest integer such that $\Sigma_{k=1}^{m}\left\|y_{n-m+k}^{k}\right\| \neq 0$ and let $p$ be the smallest integer such that $y_{n-m+p}^{p} \neq 0$. Because we have

$$
\sum_{k=p}^{m} T^{k-1} y_{n-m+k}^{k}=-\sum_{j=m+1}^{n} \sum_{k=1}^{j} T^{k-1} y_{n-j+k}^{k} \text { in } \operatorname{ker} T^{n-m},
$$

it follows that $y_{n-m+p}^{p}+\cdots+T^{m-p} y_{n}^{m}$ is in ker $T^{n-m+p-1}$. If we let $P$ denote the orthogonal projection of $H$ onto $X_{n-m+p}$, then

$$
\begin{aligned}
y_{n-m+p}^{p}+P\left(T y_{n-m+p+1}^{p+1}+\cdots+T^{m-p} y_{n}^{m}\right) \\
=P\left(y_{n-m+p}^{p}+\cdots+T^{m-p} y_{n}^{m}\right)=0
\end{aligned}
$$

since $X_{n-m+p}$ is orthogonal to $\operatorname{ker} T^{n-m+p-1}$ and $y_{n-m+p}^{p}$ is in $X_{n-m+p}$. Moreover, since $y_{n-m+p}^{p}$ is orthogonal to $T^{m-p} y_{n}+T^{m-p-1} y_{n-1}+\cdots+$ $T Y_{n-p+m+1}$, it follows that

$$
y_{n-m+p}^{p} \perp P\left(T y_{n-m+p+1}^{p+1}+\cdots+T^{m-p} y_{n}^{m}\right)
$$

and hence that $y_{n-m+p}^{p}=0$ which is a contradiction.

This completes the proof of the lemma.

3. Proof of Theorem 1. By applying Lemma 2 to $T$ and $T^{*}$ we obtain quasi-affinities $X$ and $X_{*}$ together with Jordan operators $T_{0}$ and $T_{1}$ such that 
$T X=X T_{0}$, and $T^{*} X_{*}=X_{*} T_{1}$. Hence, $X_{*}^{*} T=T_{1}^{*} X_{*}^{*}$ and $T$ is a quasi-affine transform of the Jordan operator $T_{1}^{*}$. Thus $T_{0}$ is a quasi-affine transform of $T_{1}^{*}$ and hence $T_{0}$ and $T_{1}^{*}$ are quasi-similar by Lemma 1 . Consequently, $T$ is a quasi-affine transform of $T_{0}$ and we have established that $T$ and $T_{0}$ are quasisimilar.

4. We make several remarks before continuing.

Since there exist quasi-nilpotent operators $T$ such that $\operatorname{ker} T=(0) \neq$ ker $T^{*}$ (for example, take $T$ to be the weighted shift with weights $1,1 / 2,1 / 3$, ... ), Theorem 2 is not valid for quasi-nilpotent operators.

As a consequence of Theorem 2, observe that Lemma 1 holds for all nilpotent operators, that is, if one nilpotent operator is a quasi-affine transform of another, then the two operators are actually quasi-similar.

Lastly, by using the Dunford-Riesz spectral decomposition Theorem 2 can be shown to hold for algebraic operators with real spectrum.

5. Theorem 1 provides a Jordan model for every nilpotent operator on Hilbert space. However, in contrast with the finite dimensional case, distinct Jordan models may be quasi-similar. Fortunately, the situation is not as complicated as it might first appear. We obtain a canonical choice and hence a complete set of quasi-similarity invariants for nilpotent operators after introducing some terminology.

For each integer $m(1 \leqslant m<\infty)$ and each infinite cardinal $\aleph$, let $J_{m}^{\aleph}$ denote the Jordan operator defined by the $m \times m$ operator matrix

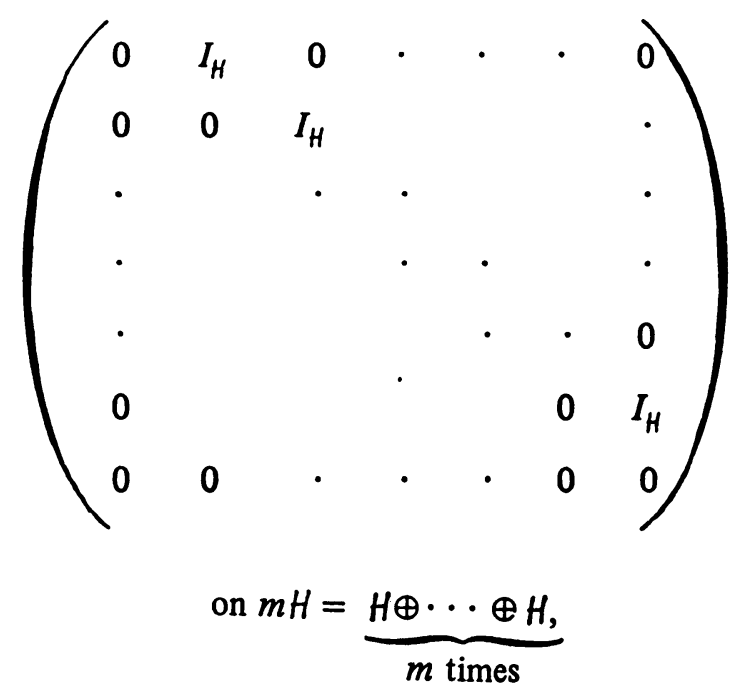

where $H$ is a Hilbert space of dimension $\aleph$.

THEOREM 3. Every nilpotent operator is quasi-similar to a unique Jordan 
model of the form $\oplus J_{m_{i}}^{\aleph_{i}} \oplus N$, where $1 \leqslant m_{1}<m_{2}<\cdots<m_{k}<\infty, \aleph_{1}>$ $\aleph_{2}>\cdots>\aleph_{k}$, and $N$ is a finite rank Jordan model $\bigoplus_{j=1}^{n} T_{j}$ on $\bigoplus_{j=1}^{n} c^{l_{j}}$ with $m_{k}<l_{j}$ for $j=1,2, \ldots, n$

Proof. By Theorem 1 we need only consider Jordan models and easy arguments reduce the result to proving that $J_{k}^{\aleph} \oplus J_{k-1}^{\aleph}$ and $J_{k}^{\aleph}$ are quasi-similar for each $1 \leqslant k<\infty$ and infinite cardinal $\aleph$. Moreover, since $J_{k}^{\aleph^{*}}$ and $J_{k-1}^{\aleph^{*}}$ are unitarily equivalent to $J_{k}^{\aleph}$ and $J_{k-1}^{\aleph}$, respectively, it is sufficient to show that $J_{k}^{\aleph}$ is a quasi-affine transform of $J_{k}^{\aleph} \oplus J_{k-1}^{\aleph}$. Let $H$ be a Hilbert space of dimension $\aleph$ and suppose $A$ and $B$ are operators on $H$ which satisfy

(1) $\operatorname{ker} A=(0)$,

(2) $\operatorname{clos}(A H)=H$, and

(3) $\operatorname{clos}\{A x \oplus B x: x \in H\}=H \oplus H$.

Then the identity

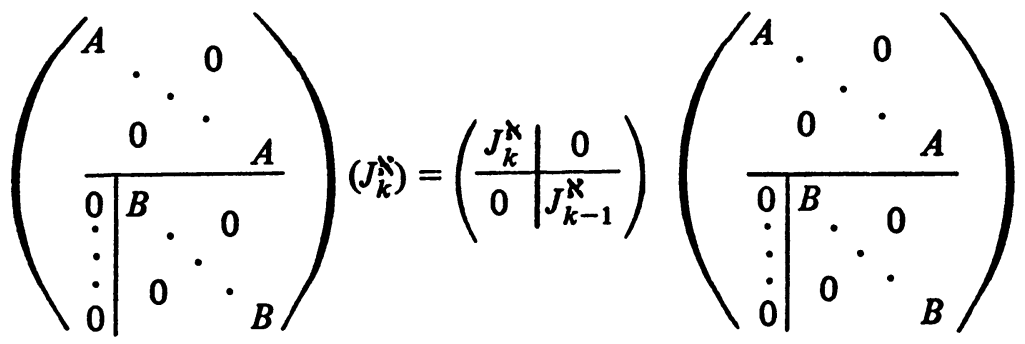

would complete the proof since (1), (2) and (3) imply that the matrix

$$
\left(\begin{array}{ccc}
A & & 0 \\
0 & \cdot & A \\
\hline \vdots & B & 0 \\
0 & 0 & \cdot \\
\hline
\end{array}\right)
$$

defines a quasi-affinity from $k H$ to $(2 k-1) H$.

There are various ways of exhibiting operators satisfying (1), (2) and (3). For example, let $M_{1}$ and $M_{2}$ denote multiplication by the characteristic functions of the first and second quarters $Q_{1}$ and $Q_{2}$ of the unit circle respectively, defined from the Hardy space $H^{2}$ to the $L^{2}$ spaces $L^{2}\left(Q_{1}\right)$ and $L^{2}\left(Q_{2}\right)$ respectively. If $V_{1}, V_{2}$, and $V_{3}$ are unitary maps from $H$ onto $H^{2} \otimes H, L^{2}\left(Q_{1}\right) \otimes H$, and $L^{2}\left(Q_{2}\right) \otimes H$, then $A=V_{2}^{*}\left(M_{1} \otimes I_{H}\right) V_{1}$ and $B=V_{3}^{*}\left(M_{2} \otimes I_{H}\right) V_{1}$ have the desired properties.

This theorem is probably indicative of the kind of uniqueness one can expect for Jordan models for $C_{0}$-operators of infinite multiplicity. 
We conclude this section with a corollary which completes the classification of nilpotents up to quasi-similarity.

COROLLARY. If $T_{1}$ and $T_{2}$ are nilpotent operators on the Hilbert spaces $H_{1}$ and $H_{2}$, respectively, then $T_{1}$ and $T_{2}$ are quasi-similar if and only if $\operatorname{dim} \operatorname{clos}\left[T_{1}^{l} H_{1}\right]=\operatorname{dim} \operatorname{clos}\left[T_{2}^{l} H_{2}\right]$ for $l=0,1, \ldots$.

Proof. If $X$ is a quasi-affinity from $H_{1}$ to $H_{2}$ such that $T_{2} X=X T_{1}$, then

$$
\operatorname{clos}\left[X T_{1}^{l} H_{1}\right]=\operatorname{clos}\left[T_{2}^{l} X H_{1}\right]=\operatorname{clos}\left[T_{2}^{l} H_{2}\right]
$$

which implies that $\operatorname{dim} \operatorname{clos}\left[T_{1}^{l} H_{1}\right]=\operatorname{dim} \operatorname{clos}\left[T_{2}^{l} H_{2}\right]$ for $l=0,1,2, \ldots$. Conversely, an easy argument shows that the Jordan model given in the theorem is uniquely determined by these dimensions.

6. The results of this note enable us to extend a characterization of reflexive operator of Deddens and Fillmore [4] to infinite dimensional spaces. Recall that a linear subspace $M$ of the Hilbert space $H$ is said to be para-closed for the operator $T$ on $H$ if $M$ is the range of some bounded operator on $H$. Let us call an operator $T$ on $H$ para-reflexive if any operator $U$ on $H$ leaving invariant the para-closed-invariant spaces of $T$ is an entire function of $T$. The definition is one of the possible natural extensions to infinite dimensional spaces of the concept of reflexive operators on a finite dimensional space.

We begin this section with a result which may have some independent interest.

Proposition 1. Para-reflexivity is preserved under quasi-similarity.

Proof. If $T$ and $S$ are quasi-similar and $S$ is para-reflexive we must show that $T$ is also para-reflexive. If $T$ is not algebraic, then by virtue of Theorem 2 [6], $T$ is para-reflexive. Thus we can assume that $T$ (and consequently $S$ also) is algebraic. Suppose $T A=A S, B T=S B$ where $A, B$ are quasi-affinities, and let $Z$ be an operator leaving invariant every finite dimensional subspace invariant for $T$, that is, for every $h$ in $H$ there exists some polynomial $p_{h}$ such that $Z h=p_{h}(T) h$. If we set $Z_{0}=B Z A$, then

$$
Z_{0} h_{0}=B Z A h_{0}=B p_{A h_{0}}(T) A h_{0}=B A p_{A h}(S) h_{0} \text { is in } B A H
$$

for every $h_{0}$ in $H$. Thus $X=(B A)^{-1} Z_{0}$ is, by the closed graph theorem, an operator on $H$ such that

$$
X h_{0} \text { is in the finite dimensional space } \bigvee_{j \geq 0} S^{\prime} h_{0}
$$

for every $h_{0}$ in $H$. It follows that $X$ leaves invariant every finite dimensional 
subspace of $H$ invariant under $S$. Thus, since $S$ is para-reflexive, we infer from Corollary 2 [6] that $X=q(S)$, where $q$ is a suitable polynomial. Consequently, $B Z A=Z_{0}=B A q(S)=B q(T) A$ and hence $Z=q(T)$. Using Corollary 2 [6] once again, we conclude that $T$ is para-reflexive.

A nilpotent operator on $H$ is said to satisfy the Deddens-Fillmore condition [4], if either $\operatorname{dim} H \leqslant 1$ or its Jordan model $\bigoplus_{\alpha \in A} J_{\alpha}$ has the following property: If $n_{\alpha}$ denotes the order of the matrix of $J_{\alpha}(\alpha \in A)$ and $\alpha_{0}$ is chosen in $A$ such that

$$
n_{\alpha_{0}}=\max \left\{n_{\alpha} \mid \alpha \in A\right\}
$$

then

$$
\max \left\{n_{\alpha} \mid \alpha \in A \backslash\left\{\alpha_{0}\right\}\right\} \geqslant n_{\alpha_{0}}-1 \text {. }
$$

Proposition 2. A nilpotent operator $T$ on $H$ is para-reflexive if and only if it satisfies the Deddens-Fillmore condition.

Proof. By virtue of Proposition 1 and Theorem 1, it is sufficient to prove the statement in case $T=\bigoplus_{\alpha \in A} J_{\alpha}$. Exactly as in [4] we can prove that if this $T$ does not fulfill the Deddens-Fillmore condition then $T$ does not have property (A) or (B) of Corollary 2 [6]. Thus, by this corollary, $T$ is not parareflexive.

Let us now show the sufficiency of the Deddens-Fillmore condition. It is clear that we can assume that

$$
T=J_{0} \oplus J_{1} \oplus\left(\bigoplus_{\alpha \in B} J_{\alpha}\right),
$$

where the order $n_{i}$ of $J_{i}$ is the maximum occurring in formula (i) above ( $i=0$, $1)$; thus the order of any $J_{\alpha}(\alpha \in B)$ is not greater than $n_{1}$. Now let $Z$ be an operator leaving invariant all para-closed subspaces invariant for $T$. Then obviously

$$
Z=Z_{0} \oplus Z_{1} \oplus\left(\bigoplus_{\alpha \in B} Z_{\alpha}\right)
$$

and for any $h=h_{0} \oplus h_{1} \oplus\left(\bigoplus_{\alpha \in B} h_{\alpha}\right)$ there exists a polynomial $p_{h}$ such that

$$
\begin{aligned}
& Z h=p_{n}(T) h \text {, that is, } \\
& \left(Z_{0} \oplus Z_{1} \oplus Z_{\alpha}\right)\left(h_{0} \oplus h_{1} \oplus h_{\alpha}\right)=p_{h}\left(J_{0} \oplus J_{1} \oplus J_{\alpha}\right)\left(h_{0} \oplus h_{1} \oplus h_{\alpha}\right)
\end{aligned}
$$

for every $\alpha$ in $B$. The above relation shows in particular that $Z_{0} \oplus Z_{1} \oplus Z_{\alpha}$ leaves invariant every invariant subspace of $J_{0} \oplus J_{1} \oplus J_{\alpha}$. By virtue of the Deddens-Fillmore theorem there exists a unique polynomial $q_{\alpha}$ of degree $\leqslant n_{0}$ such that 
such that

$$
\begin{aligned}
Z_{0} \oplus Z_{1} \oplus Z_{\alpha} & =q_{\alpha}\left(J_{0} \oplus J_{1} \oplus J_{\alpha}\right) \\
& =q_{\alpha}\left(J_{0}\right) \oplus q_{\alpha}\left(J_{1}\right) \oplus q_{\alpha}\left(J_{\alpha}\right),
\end{aligned}
$$

for every $\alpha$ in $B$. Thus for $\alpha, \beta$ in $B$ we have

$$
q_{\alpha}\left(J_{0}\right)=Z_{0}=q_{\beta}\left(J_{0}\right) \text {. }
$$

Since $J_{0}$ is of order $n_{0}$ and $q_{\alpha}, q_{\beta}$ are of degree $\leqslant n_{0}$, (3) implies $q_{\alpha}=q_{\beta}$. Consequently, there exists a polynomial $q$ of degree $\leqslant n_{0}$ such that $q_{\alpha} \equiv q$ for every $\alpha$ in $B$. From (2) we infer

$$
Z=Z_{0} \oplus Z_{1} \oplus\left(\bigoplus_{\alpha \in B} Z_{\alpha}\right)=q\left(J_{0}\right) \oplus q\left(J_{1}\right) \oplus\left(\bigoplus_{\alpha \in B} q\left(J_{\alpha}\right)\right)=q(T)
$$

which finishes our proof.

THEOREM 4. An operator $T$ is para-reflexive if and only if either it is nonalgebraic or it is algebraic and the nilpotents corresponding to the points of the spectrum of $T$ satisfy the Deddens-Fillmore condition.

Proof. This follows at once from Proposition 2 above, the DunfordRiesz spectral decomposition of an algebraic operator, and Corollary 1.

\section{REFERENCES}

1. H. Bercovici, On the Jordan model of nilpotent operators, Private communication.

2. H. Bercovici, C. Foiaş and B. Sz.-Nagy, Compléments à l'étude des opérateurs de classe $C_{0}$. III, Acta Sci. Math. (Szeged) 37 (1975), 313-322.

3. F. F. Bonsall and P. Rosenthal, Certain Jordan operator algebras and double commutant theorems (to appear).

4. J. A. Deddens and P. A. Fillmore, Reflexive linear transformations, Linear Algebra and Appl. 10 (1975), 89-93. MR 50 \#10856.

5. R. G. Douglas, Banach algebra techniques in operator theory, Academic Press, New York, 1972. MR 50 \#14335.

6. R. G. Douglas and C. Foiaş, Infinite dimensional versions of a theorem of Brickman-Fillmore, Indiana Univ. Math. J. 25 (1976), 315-320.

7. P. Rosenthal and H. Radjavi, Invariant subspaces, Springer-Verlag, Berlin and New York, 1974.

8. B. Sz.-Nagy and C. Foias, Harmonic analysis of operators on Hilbert space, Akad. Kiadó, Budapest, 1967; English rev. transl., North-Holland, Amsterdam; American Elsevier, New York; Akad. Kiadó, Budapest, 1970. MR 37 \#778; 43 \#947.

\footnotetext{
INSTITUTUL NATIONAL PENTRU CREAŢIE ŞTINŢIFICĂ ŞI TEHNICA, BUCURESTI, RUMANIA
}

DEPARTMENT OF MATHEMATICS, STATE UNIVERSITY OF NEW YORK, STONY BROOK, NEW YORK 11794 RUMANIA

DEPARTMENT OF MATHEMATICS, UNIVERSITY OF BUCHAREST, BUCHAREST, 